\title{
Decision making across social contexts: competition increases preferences for risk in chimpanzees and bonobos
}

\author{
Alexandra G. Rosati*, Brian Hare \\ Department of Evolutionary Anthropology, Center for Cognitive Neuroscience, Duke University, Durham, NC, U.S.A.
}

\section{A R T I C L E I N F O}

\section{Article history:}

Received 17 February 2012

Initial acceptance 23 March 2012

Final acceptance 27 June 2012

Available online $\mathrm{xxx}$

MS. number: A12-00143R

\section{Keywords:}

ape

competition

decision making

emotion

risk

social context
Context can have a powerful influence on decision-making strategies in humans. In particular, people sometimes shift their economic preferences depending on the broader social context, such as the presence of potential competitors or mating partners. Despite the important role of competition in primate conspecific interactions, as well as evidence that competitive social contexts impact primates' social cognitive skills, there has been little study of how social context influences the strategies that nonhumans show when making decisions about the value of resources. Here we investigate the impact of social context on preferences for risk (variability in payoffs) in our two closest phylogenetic relatives, chimpanzees, Pan troglodytes, and bonobos, Pan paniscus. In a first study, we examine the impact of competition on patterns of risky choice. In a second study, we examine whether a positive play context affects risky choices. We find that (1) apes are more likely to choose the risky option when making decisions in a competitive context; and (2) the play context did not influence their risk preferences. Overall these results suggest that some types of social contexts can shift patterns of decision making in nonhuman apes, much like in humans. Comparative studies of chimpanzees and bonobos can therefore help illuminate the evolutionary processes shaping human economic behaviour.

(c) 2012 The Association for the Study of Animal Behaviour. Published by Elsevier Ltd. All rights reserved.
Human economic decision making is marked by systematic biases. Context can have a profound effect on the preferences that people show: framing (or presentation of the choice), the presence of irrelevant alternatives and previous experiences all influence how people make decisions, even when rational choice theory predicts it should not (Kahneman \& Tversky 2000). Contextual factors that are external to the choice at hand can have similar effects: levels of sleep deprivation (Reynolds \& Schiffbauer 2004; Glass et al. 2011; Venkatraman et al. 2011), mood or emotional state (Raghunathan \& Pham 1999; Lerner \& Keltner 2001; Fessler et al. 2004) and stress level (Bault et al. 2008; Kassam et al. 2009; Porcelli \& Delgado 2009) can all shift strategies. Finally, social context, the presence or absence of particular social partners, can affect preferences in several situations, including decisions about time and risk (Wilson \& Daly 2004; Bault et al. 2008; Ermer et al. 2008; Hill \& Buss 2010). Understanding the role of social context in shaping decision-making strategies is particularly important for biologists and psychologists who are interested in understanding behaviour in the real world, as gregarious species like primates

* Correspondence: A. G. Rosati, Department of Evolutionary Anthropology, Center for Cognitive Neuroscience, Box 90383, Biological Science Building, Duke University, NC 27708, U.S.A.

E-mail address: alexandra.rosati@duke.edu (A. G. Rosati). must constantly make value-based decisions in the company of others. While cooperation in nonhumans has received increasing attention, little comparative research has examined the role of social context in shaping decisions when an individual's payoffs do not directly depend on their partner's behaviour. In the current study we therefore examine the impact of social context on risk preferences in chimpanzees, Pan troglodytes, and bonobos, Pan paniscus, our closest phylogenetic relatives.

Studies of social context and decision making in humans have generally been motivated by evolutionary hypotheses concerning how the presence or absence of certain social partners might alter how decision makers value resources. In one study, Wilson \& Daly (2004) found that men who viewed images of attractive women had steeper temporal discounting rates, devaluing the future much more heavily than men who viewed unattractive women or cars. That is, looking at attractive women made men more impulsive or present oriented, possibly because the attractive women cued men into the possibility that current possession of goods could increase mating opportunities. Social context also modulates people's willingness to take economic risks (or accept variability in payoffs). For example, men became more likely to choose risky options when their choices were watched by an observer of equal status, compared to when relative status was more skewed (Ermer et al. 2008). Similarly, people were more likely to make risky choices if doing so might render them comparatively better off than 
a hypothetical competitor (Hill \& Buss 2010). Finally, social context can affect the subjective utility that people experience after receiving different risk outcomes. In particular, while people weighted the threat of loss more heavily when making decisions in private, they focused on the possibility of winning more than they did other outcomes when making decisions in public (Bault et al. 2008). Together, these results indicate that people may show more risk-prone strategies when doing so allows people to outcompete others. More generally, these types of studies indicate that social context can influence economic decisions that involve individual-level strategies (unrelated to social interactions per se), possibly because social context can be an important cue as to the value of various resources in the current environment.

Comparative studies have revealed that animals also show context-sensitive choice in many situations (reviewed in Rosati \& Stevens 2009). Contextual aspects of the decision, including framing and the set of available options, can shift nonhuman preferences much like in humans (e.g. capuchins, Cebus apella: Lakshminarayanan et al. 2011; starlings, Sturnus vulgaris: Marsh \& Kacelnik 2002; honeybees, Apis mellifera: Shafir et al. 2002; hoarding grey jays, Perisoreus canadensis: Waite 2001). Internal states such as satiation level and energy budget can also alter animal strategies in potentially adaptive ways (e.g. dark-eyes juncos, Junco hyemalis: Caraco 1981; chimpanzees: Gilby \& Wrangham 2007; starlings: Schuck-Paim et al. 2004). However, there is less evidence bearing on whether social context can alter economic preferences. The presence of others does influence more naturalistic foraging behaviours such as caching that involve decisions about resources (Clayton et al. 2007); for example, both scrub-jays, Aphelocoma californica, and ravens, Corvus corax, account for the presence of potential competitors when deciding where to cache (Emery \& Clayton 2001; Dally et al. 2004, 2006; Bugnyar \& Heinrich 2005; Bugnyar et al. 2007). In addition, the characteristics that partners show are clearly important for cooperative behaviours that are intrinsically social in nature, both in primates (Werdenich \& Huber 2002; Melis et al. 2006b, c; Hare et al. 2007; Yamamoto \& Tanaka 2009) as well as corvids such as rooks, Corvus frugilegus (Seed et al. 2008). However, to our knowledge there has been no study examining how social context mediates individual strategies for economic decision making in animals. Importantly, many of the social cues that influence human decision making should also be important for other species that face similar challenges in acquiring resources.

Competition may be especially relevant to understanding nonhuman primate decision making, as most primates live in groups. While gregariousness has many advantages, groupmates are also competitors for resources such as food or mates, and consequently, the social lives of most primates are dominated by intense competition with conspecifics (Wrangham 1980; van Schaik \& van Hooff 1983; Sterck et al. 1997). Indeed, observations from the wild suggest that competition is an important component of social interactions in chimpanzees and bonobos in particular. In terms of intragroup competition, both species compete over monopolizable food such as meat or large fruits (Boesch et al. 2002). Both species also compete for dominance rank, and rank can play a crucial role in determining who has preferential access to food and mates in social interactions (Parish 1996; Pusey et al. 1997; Constable et al. 2001; Wittig \& Boesch 2003a; Surbeck et al. 2011). Various aspects of intergroup behaviour in apes are likely also related to competition for resources. For example, chimpanzee groups can expand their territories, and consequently gain access to more food, by engaging in boundary patrols involving lethal aggression directed towards foreign chimpanzees (Mitani et al. 2010). Overall, this suggests that competition is important in shaping nonhuman apes' decisions about resources in natural contexts, much like the results from human experimental studies (Bault et al. 2008; Ermer et al. 2008; Hill \& Buss 2010).

Indeed, the importance of competition in primate social behaviour is underscored by many theoretical accounts suggesting that the evolution of primate intelligence may be best understood by examining complex social interactions. Many of these accounts focus on forms of competition, such as political manoeuvring (de Waal 1982) or 'Machiavellian' intelligence (Byrne \& Whiten 1988). Along these lines, experimental studies indicate that competitive contexts play an important role in shaping primate social behaviour and cognition (Hare 2001). For example, apes are more skilled at comprehending and responding appropriately to human's communicative gestures in competitive contexts, compared to matched situations involving cooperative motives (Hare \& Tomasello 2004; Herrmann \& Tomasello 2006). Indeed, many species, including apes (Tomasello et al. 2003; Hare \& Tomasello 2004; Hare et al. 2006; Kaminski et al. 2008), monkeys (marmosets, Callithrix jacchus: Burkhart \& Heschl 2007; rhesus macaques, Macaca mulatta: Flombaum \& Santos 2005; capuchins: Hare et al. 2003), and ringtailed lemurs, Lemur catta (Sandel et al. 2011), show complex social cognitive abilities when competing with conspecifics or humans, using information about the gaze direction or even visual perceptions of others. Other taxonomic groups that also face competition with conspecifics, such as corvids, show similar skills when caching in competitive contexts, as discussed previously (Emery \& Clayton 2001; Dally et al. 2004, 2006; Bugnyar \& Heinrich 2005; Bugnyar et al. 2007). Yet despite the extensive evidence that competition it important for social cognition, the impact of competitive contexts on other domains such as decision making has been largely unexplored. In the current study we therefore sought to examine how competition influences risky decision making in chimpanzees and bonobos. Such comparative studies can illuminate the evolutionary origins of human-like choice patterns that account for broader social context when assessing the value of resources.

We tested a sample of semi-free ranging, wild-born chimpanzees and bonobos on a risk task involving variation in food quality. In study 1, we compared risk preferences following a competitive interaction, versus a neutral context. In study 2 , we examined whether social interactions more generally influence risk preferences by comparing choices in a positive context to a neutral context. We predicted that chimpanzees would choose the risky option more than bonobos overall, following previous work with these species (Heilbronner et al. 2008; Haun et al. 2011; Rosati \& Hare 2011). We also predicted that if competition increases riskprone decision making in apes, then apes should show a greater propensity to choose the risky option when faced with competition, compared to a neutral context. Finally, we predicted that if any social interaction shifts risk preferences, then apes should show different patterns of choice in a play context, relative to a neutral context. In contrast, if competitive contexts are especially relevant to apes, then the play context might not influence risk preferences.

\section{STUDY 1: DOES COMPETITION INFLUENCE APE RISK PREFERENCES?}

We examined the willingness of chimpanzees and bonobos to accept variability in payoffs to acquire better food items. Here, apes chose between a risky option that provided either a good (preferred) or a bad (nonpreferred) food type with equal probability, and a safe option that always delivered an intermediately preferred food type (following the general methods in Rosati \& Hare 2011). We confirmed that apes modulated their choices according to the relative value of the risky and safe options by varying whether the safe option provided one or three pieces of the 
intermediately preferred food across trials (with amount always known prior to their choice). Finally, we assessed how competition affected the apes' decisions by comparing risk preferences in a competitive context where a human competed with the ape over food, versus a neutral context where the human was present but did not interact socially with the ape. We used a human social partner (as opposed to a conspecific) following multiple studies examining responses to competition in primates (Hare \& Tomasello 2004; Flombaum \& Santos 2005; Hare et al. 2006; Melis et al. 2006a; Santos et al. 2006; Sandel et al. 2011). In particular, this method allowed us to examine the apes' responses to a competitor showing predetermined behavioural patterns, therefore equating the competitor's actions across subjects.

\section{Methods}

\section{Ethics statement}

All behavioural studies were noninvasive. The studies had approval from the Institutional Animal Care and Use Committee of Duke University (protocol number A078-08-03) and strictly adhered to the legal requirements of the countries in which they were conducted. Animal husbandry and research practices complied with the PASA Primate Veterinary Healthcare Manual and the policies of Tchimpounga Chimpanzee Sanctuary in Pointe Noire, Republic of Congo and Lola ya Bonobo Sanctuary in Kinshasa, Democratic Republic of Congo. All apes at both sites were socially housed, and the vast majority semi-free-ranged in large tracts of tropical forest during the day (5-40 ha across groups). In the evening, all apes spent the night in indoor dormitories $\left(12-160 \mathrm{~m}^{2}\right)$. Apes were tested individually in these familiar dormitories buildings. Following testing, most apes were released back with their larger social group outside. Apes had ad libitum access to water and were never food deprived for testing. In addition to the food the apes could eat in their forest enclosures, they were fed a variety of fruits and vegetables and other speciesappropriate food two to four times daily. Subjects completed no more than one test session per day, and all tests were voluntary: if the ape stopped participating, the session was halted.

\section{Subjects}

We tested 36 apes (see Supplementary Table S1 for all individual subject details): 20 chimpanzees from Tchimpounga Chimpanzee Sanctuary (7 females and 13 males; mean age 11.3 years; range $7-21$ years) and 16 bonobos from Lola ya Bonobo Sanctuary (5 females and 11 males; mean age 8.4 years; range $6-12$ years). Apes in African sanctuaries are typically born in the wild, and enter the sanctuary after being confiscated at an early age ( $\sim 2-3$ years old) as a result of the trade in apes for pets and bushmeat. Previous work indicates that sanctuary apes are psychologically healthy relative to other captive populations (Wobber \& Hare 2011). All apes were familiar with the basic set-up involving entering a testing room, interacting with a human experimenter and making dichotomous choices between two options, as they had previously participated in a large battery involving a variety of cognitive tasks including numerical discrimination, use of social cues and spatial representations (Herrmann et al. 2007, 2010). Thirteen chimpanzees and 10 bonobos had participated in a previous risk study using the basic task approximately 1 year before (A. G. Rosati \& B. Hare, unpublished data), whereas the rest were naïve to the risky choice procedure (see Table S1). None of the individuals had previously experienced competitive interactions during this risk task.

\section{General set-up and risky choice task}

Apes completed a total of four sessions in the following order: a food preference pretest to identify appropriate food types for use in the task; a risk introduction session to familiarize the apes with the basic set-up; and two test sessions (the competition condition and neutral condition, in counterbalanced order across subjects). The methods followed those of previous studies using the qualitative risk task (e.g. Rosati \& Hare 2011).

In trials, one experimenter and the ape sat across from each other at a table $(80 \mathrm{~cm}$ wide, $40 \mathrm{~cm}$ deep, $50 \mathrm{~cm}$ tall) with a sliding top, separated by wire mesh or bars (see Supplementary Fig. S1 for photos of the task). Subjects first saw the experimenter place the intermediately preferred food type (the safe option) on one side of the table, and then cover it with an overturned blue bowl $(17.5 \mathrm{~cm}$ in diameter, $5.5 \mathrm{~cm}$ tall). Next, the experimenter placed an identical, but empty, overturned bowl on the other side of the table (the risky option). The experimenter next occluded only the risky option with a small freestanding occluder ( $40.5 \mathrm{~cm}$ wide, $24 \mathrm{~cm}$ deep, $24 \mathrm{~cm}$ tall). The experimenter then showed the subject the 'risk outcome' container (a red bowl) that contained the set of food types that were possible risk outcomes for that trial. That is, in a normal risky choice trial, the container would have both the good and the bad food types. The experimenter then moved the risk outcome container behind the occluder and placed just one of those food items under the risk bowl. The risky option therefore contained only one of the possible outcomes that the subject had previously seen in the risk outcome container, but apes did not know which it would be before they made a choice. Finally, the experimenter touched both cups simultaneously, while also lifting up the safe bowl to remind the subject of the safe option's value. Consequently, subjects always knew what they would receive from the safe option, but did not know whether they would receive a good or bad outcome from the risky option. Finally, the experimenter pushed the table forward so that the ape could choose one of the options. At the time of choice, the experimenter looked at the middle of the table so as to not cue the apes. If the ape chose the safe option, the experimenter did not reveal the contents of the risky option.

There was a $30 \mathrm{~s}$ intertrial interval (ITI) between trials (timed with a stopwatch), starting when subjects put the last piece of food in their mouth. Subjects had $20 \mathrm{~s}$ to choose once the table was pushed forward; if they failed to choose in this period, the trial was repeated at the end of the session. If subjects protruded both hands simultaneously in the task (e.g. tried to choose both options at once), the experimenter pulled the table back and re-presented it after a few seconds until the subjects made a discrete choice. If apes did not participate for three trials in the session, it was stopped and repeated the next day.

\section{Food preference pretest}

In the initial food preference session, we examined apes' preferences across three categories of food: highly preferred (chimpanzees: banana slice; bonobos: apple cube), intermediately preferred (peanuts halves for both species) and nonpreferred (chimpanzees: cucumber slice; bonobos: lettuce leaf), following previous studies (Rosati \& Hare 2011). Food types differed based on availability at the two sanctuaries. Apes first completed six 'introduction trials' where the experimenter placed only one piece of food on the table, to ensure that the apes would point or reach towards the food. They then completed 18 'preference trials'. Here, the experimenter first placed two different pieces of food on opposite sides of the table; subjects could view them for $4 \mathrm{~s}$. The experimenter then covered them with identical bowls (to introduce this aspect of the risk task) and pushed the table forward so the ape could choose. Apes chose between each possible pairing of the three food types a total of six times, with side assignment for the food counterbalanced across trials. Based on their preferences in the pretest, the most preferred food was assigned as the good risk outcome, the least preferred food as the bad risk outcome, and the intermediate food as the safe option. 


\section{Risk introduction session and control trials}

Prior to completing the main test sessions, all apes completed a 'risk introduction session' consisting of 12 exposure trials and 12 control trials. In exposure trials, apes saw the experimenter demonstrate the procedure for only one option (e.g. either the risk option or the safe option). Subjects thus gained experience with the outcomes of both the safe and risky options, before making choices between them in the main task. Six of the exposure trials involved the risky option (half providing a good outcome and half providing a bad outcome) and six involved the safe option (with half providing one piece of the safe food and half providing three pieces). The safe and risk option trials were randomly intermixed, with side assignment counterbalanced. Subjects then completed 12 control trials (following the procedure in Rosati \& Hare 2011). These trials were similar to the risk trials in terms of their attention and memory demands, but the rewards varied to assess the apes' comprehension of the task. As in the risky choice trials, subjects always received only one of the possible outcomes from the risky option. Subjects completed three types of control trials in the introduction, and two additional types were interspersed in test sessions (see Supplementary Fig. S2 for a diagram of all control trial types). When describing control trial types, we refer to the different options using the same label used in the main task since the structural procedure of the task was the same, even though the reward contingencies differed here.

Inhibition (four trials, introduction session). These trials proceeded as normal risky choice trials, but in a final step the experimenter removed the food from under the safe option. If subjects could inhibit reaching for the last location where they saw food (because it was subsequently removed), they should have chosen the risky option.

Comprehension 1 (four trials, introduction session). Here, the safe option provided two pieces of a preferred food, and subjects saw two identical pieces in the risk outcome container. If subjects understood that the risky option only provides one of the possible outcomes that they previously saw in the outcome container, they should have chosen the safe option. That is, even though the same amount of food was initially present in both the safe bowl and the risky outcome container, the safe option would ultimately provide more food.

Comprehension 2 (four trials, introduction session). The safe option provided a small piece of a preferred food, and the risky outcome container contained two larger pieces of the preferred food. If subjects actively compared the potential rewards they could receive from the safe and risky options, they should have preferred the risky option because it would deliver a bigger piece of food.

Attention 1 (two trials in each test session). These trials were interspersed within test sessions to confirm that apes attended to the available rewards on a trial-by-trial basis. In these trials, the safe option provided one piece of a preferred food, and subjects saw two pieces of a nonpreferred food in the risky outcome container. Subjects should have chosen the safe option because it provided the preferred food type.

Attention 2 (two trials in each test session). The safe option was the nonpreferred food, and the risk outcome bowl contained two pieces of preferred food. Subjects should have chosen the risky option because it provided the preferred food type.

\section{Test sessions and competition procedure}

Each test session involved 20 trials (16 choice trials and four randomly interspersed attention control trials, described previously). Apes completed two conditions (competition and neutral) in counterbalanced order across subjects. To create a competitive context, we modified a procedure used in several previous studies (Hare et al. 2006; Herrmann \& Tomasello 2006; Melis et al. 2006a;
Sandel et al. 2011). In particular, apes were exposed to a human competitor who placed food within the reach of the ape but then pulled it away when the ape approached. Previous research indicates that apes attribute different intentions to humans who tease them with food (Call et al. 2004). In addition, they modulate their willingness to delay gratification according to whether the provider was previously stingy or reliable (Stevens et al. 2011). Consequently, we did not want to conflate the person who competed with the ape and the person who conducted the main task. Consequently, experimenter one (E1) always conducted the risky choice task; experimenter two (E2) either competed with the ape (in the competition condition) or was merely present (in the neutral condition). Importantly, E1 and E2 were the same individuals across conditions for all subjects, so apes never competed with the experimenter who conducted the main risk task. That is, the same people played the roles of E1 and E2 across both conditions, to hold constant the characteristics of the interacting experimenter for all apes.

We structured the session into two halves. At the beginning of each session (prior to the start of the risk task), there was a 2 min exposure phase where E2 sat at the testing table. In the competition condition, during this time E2 repeatedly offered the ape a large piece of food. If the ape approached and attempted to take it, E2 pulled it out of reach while making an effortful grunting noise (saying 'Uh!'). In the neutral condition, E2 sat in the same position, but looked down and did not interact with the ape. Here, the food used in the competition condition was visible next to the testing table, but E2 did not touch it. Midway through the session, E2 returned to the table for a second 2 min exposure phase. E1 was not in view during either exposure phase.

After each exposure phase, E1 sat at the table and conducted the main risk task. During this time, E2 stood next to the table. During the $30 \mathrm{~s}$ ITI between trials (after the ape had received the previous choice and was waiting to initiate the next trial), E2 interacted appropriately with the ape (either continuing to offer food in the competition condition, or doing nothing in the neutral condition). At the start of each risk trial, E2 always stopped interacting with the ape and did not resume until the ape had received that trial's outcome from E1.

\section{Coding and data analyses}

Choices were coded live by E1; a second coder blind to the side assignment of the two options coded $20 \%$ of trials from video with perfect reliability (Cohen's kappa $=1.0$ ). We analysed the main choice data using two statistical approaches. The first approach used repeated measures ANOVA. For these analyses, mean proportions of choices for the risky option were calculated for each subject across both conditions and safe option value. Choice percentages in the risk task were arcsine square-root transformed to normalize the data for parametric statistics.

Second, we used generalized linear mixed models (GLMM) to analyse choices as a binary outcome variable, while accounting for correlation in responses due to repeated measures within subjects (Baayen 2008). We used the lme4 software package (Bates 2010) in the statistics program R (R Development Core Team 2011). The advantage of using a GLMM model, compared to more traditional ANOVAs, is that it allowed trial-by-trial modelling of responses to assess the importance of fixed factors, while also controlling for various covariates and random effects (such as subject). We used likelihood ratio tests to compare fit across models incorporating different factors (Bolker et al. 2008).

Across models, we included subject as random factor and assessed the effects of safe value (one or three pieces), species (chimpanzee or bonobo) and condition (competition or neutral) on choice for risky versus safe as the binary outcome variable. We also 
included trials (within a session: $1-16$ ) as a covariate. Finally, we included an index of each individual's relative preference for the various food types used in the task as a covariate to control for differences in food preferences across subjects (see below). To index a given individual's relative food preference, we took the data from that individual's food preference pretest and averaged the data across all trials where they chose between the safe option and one of the risky outcomes (good or bad). Thus, an individual that showed completely consistent ordinal preferences across the good risk outcome, the safe option and the bad risk option (e.g. always chose the good outcome over the safe option and never chose the bad outcome over the safe option) would have a mean score of $50 \%$ (reflecting that the value of the safe food type was equal to the 'average' value of the risky option). In contrast, an individual who sometimes chose the safe option over the good outcome in the pretest would have a lower score, and an individual who sometimes chose the bad risky outcome over the safe option would have a higher score.

\section{Results and Discussion}

\section{Food preference pretest}

To ensure that species did not differ in their preferences for each food category, we compared the two species' choices in the preference test. Apes chose the highly preferred food on a mean $\pm \mathrm{SE}$ of $91.7 \pm 1.9 \%$ of all available trials, the intermediately preferred food on $53.8 \pm 2.7 \%$ of trials and the nonpreferred food on $4.4 \pm 1.4 \%$ of trials. There were no species differences for any food type (independent-samples $t$ test: $P>0.33$ in all cases). In addition, we examined only those trials involving a choice between the intermediately preferred food type (e.g. the safe option in the main task) and one of the possible risk outcomes, as this best reflected the contrast apes faced in test sessions. The two species did not differ in their relative preferences for the good risk outcomes when pitted against the safe option $\left(t_{34}=-0.15, P=0.88\right)$, nor for the bad outcome when pitted against the safe option $\left(t_{34}=-0.81\right.$, $P=0.42$ ). This indicates that we were able to successfully select food items of approximately equal value for the two species.

\section{Control trials and reward history}

We next examined performance on control trials and overall reward history to assess whether either of these factors might influence our main results. Collapsing across control types, chimpanzees chose correctly on $93.8 \pm 1.8 \%$ of trials and bonobos chose correctly on $93.4 \pm 1.7 \%$ of trials. As control trial performance was highly skewed (approaching ceiling levels of performance), we used nonparametric statistics to examine species performance and found no difference overall (Mann-Whitney $U$ test: $Z=-0.60$, $\left.N_{1}=20, N_{2}=16, P=0.58\right)$. Apes were also successful on all individual control types, with no species differences (see Table 1). In addition, we assessed whether apes were distracted by the social interaction in the competition condition, as they may have had difficulty attending to the main task simultaneously. An examination of the attention controls (which were interspersed within test sessions) indicated that apes were successful in both conditions (>95\% correct), with no difference between sessions (Wilcoxon signed-ranks test: $T=4, N=36$, ties $=30, Z=-0.82, P=0.41$ ). Importantly, these trials required apes to attend to the available rewards on a trial-by-trial basis and flexibly shift their behaviour, so it is unlikely that the apes were unduly distracted in the competition condition.

We examined reward history by comparing the percentage of trials where the apes received the good outcome from the risky option. Overall, apes received the good outcome on $51.3 \pm 1.0 \%$ of trials, which did not differ from chance (one-sample $t$ test:
Table 1

Mean percentage of trials \pm SE that chimpanzees and bonobos chose correctly during control trials in the introductory session and during test sessions for study 1 (competition) and study 2 (play)

\begin{tabular}{lrrr}
\hline & Chimpanzees & Bonobos & $P$ \\
\hline Introductory session & $97.5 \pm 1.7 \%$ & $93.8 \pm 4.2 \%$ & 0.86 \\
Inhibitory & $88.8 \pm 4.2 \%$ & $93.8 \pm 3.6 \%$ & 0.54 \\
Comprehension 1 & $88.8 \pm 4.6 \%$ & $87.5 \pm 4.6 \%$ & 0.74 \\
Comprehension 2 & & & \\
Study 1 test sessions & $96.3 \pm 2.7 \%$ & $95.3 \pm 2.5 \%$ & 0.69 \\
Attention 1 & $97.5 \pm 2.5 \%$ & $96.9 \pm 2.1 \%$ & 0.74 \\
Attention 2 & & & \\
Study 2 test sessions & $96.3 \pm 2.0 \%$ & $98.2 \pm 1.8 \%$ & 0.72 \\
Attention 1 & $100.0 \pm 0.0 \%$ & $98.2 \pm 1.8 \%$ & 0.74 \\
Attention 2 & & & \\
\hline
\end{tabular}

Significance of $P$ value indicates whether the species differed in their performance in that control trial type (Mann-Whitney $U$ test).

$\left.t_{35}=1.31, P=0.20\right)$. This indicates that the apes could not detect whether they would receive the good or the bad outcome from the risky option using other cues (such as smell). There was also no effect of either species or condition on percentage of trials where apes received the good outcome ( $P>0.77$, for both cases). That is, differences in reward history cannot account for our results.

\section{Risk preferences}

We first examined overall choices for the risky option in the two species (see Fig. 1a). Collapsing across all choice trials, the chimpanzees chose the risky option on $75.6 \pm 3.9 \%$ of trials, significantly above chance (one-sample $t$ test: $t_{19}=6.59, P<0.001$, two tailed), whereas the bonobos chose the risky option on only $52.7 \pm 4.9 \%$ of trials $\left(t_{15}=0.56, P=0.58\right)$. Examining the apes' responses to the social context manipulation revealed that 19 individuals increased their choice for the risky option in the competition condition compared to the neutral condition (Wilcoxon signed-ranks test: $T=19, N=36,8$ ties, $Z=-2.661, P<0.01$; see Supplementary Table $S 1$ for individual data). A repeated measures ANOVA with condition and safe value as within-subjects factors and species as a between-subjects factor revealed a main effect of species $\left(F_{1,34}=13.11, P=0.001\right)$, with chimpanzees being more likely to choose the risky option than bonobos. In addition, apes modulated their choices for the risky option according to the value of the safe alternative $\left(F_{1,34}=75.23, P<0.001\right)$, choosing the risky option more when the safe options provided less food. Finally, there was a main effect of condition $\left(F_{1,34}=10.97, P<0.005\right)$ : while apes chose the risky option overall only on $59.4 \pm 4.6 \%$ of trials in the neutral condition, they chose it on $71.5 \pm 3.5 \%$ in the competition condition (see Fig. 1b). There were no significant interactions, indicating that both species responded similarly to the competitive context. Indeed, analysing each species separately revealed that both species independently showed increases in risky choices in the competition condition (chimpanzees: $F_{1,19}=4.97, P<0.05$; bonobos: $\left.F_{1,15}=5.72, P<0.05\right)$.

The results from the GLMM, where we modelled each individual's choice as a binary outcome, confirmed these results. We first fitted a basic model including subject as a random factor, safe value as a fixed factor, and trial (1-16) and relative food preference as covariates. Including trial number as a covariate allowed us to assess whether apes showed learning or satiation effects within the session. Although there was no overall difference between the two species in their average preference for the food types used in the task (as previously reported), including relative food preference as a covariate allowed us to control for potential individual variation in preference when modelling the apes' choices. Guided by our main hypotheses about the influence of competition and species on an individual's propensity to choose the risky option, we then 

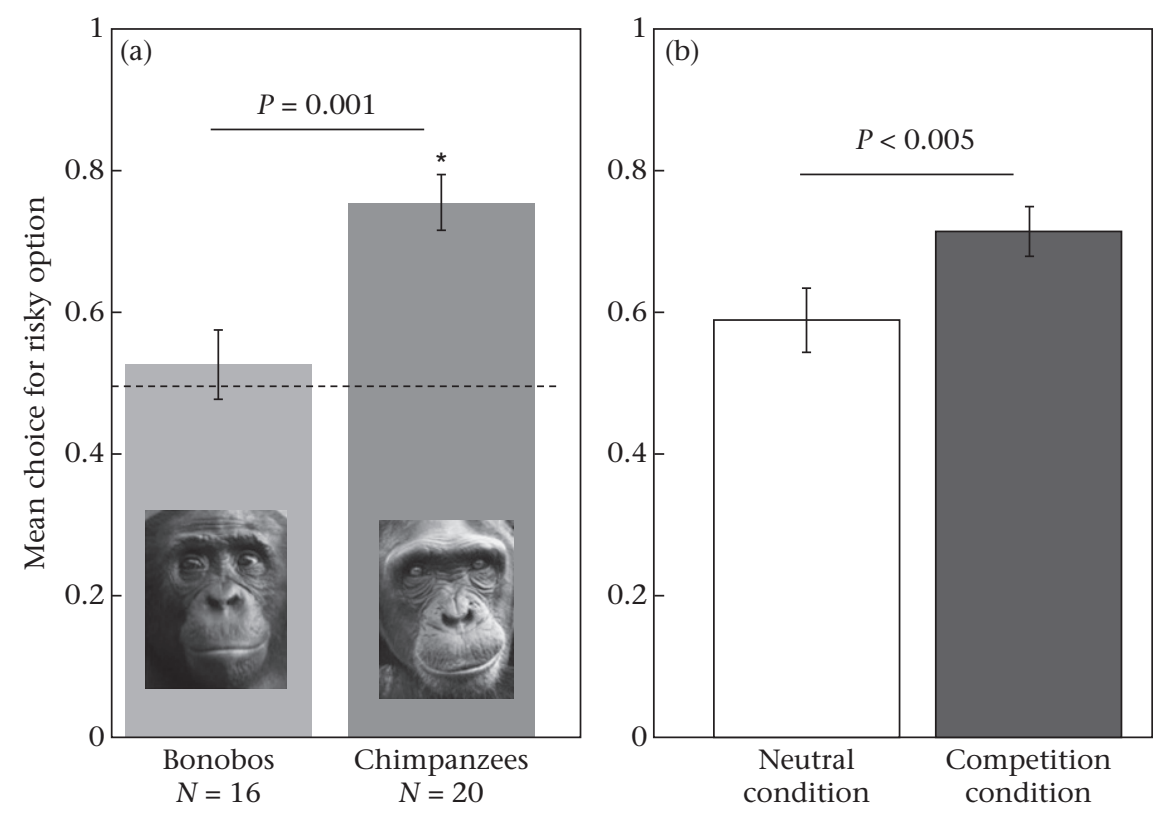

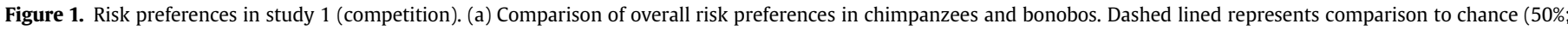

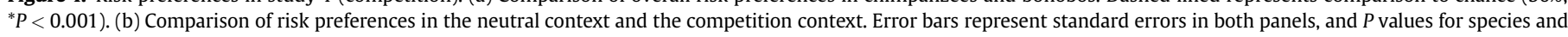
condition comparisons are based on the repeated measures ANOVA.

added these terms to the more parsimonious basic model to assess their importance. Comparing the basic model to a second model including species as an additional factor revealed a significant increase in model fit (likelihood ratio test comparing basic to species model: $\left.\chi_{1}^{2}=11.82, P<0.001\right)$. A full model including both condition and species further increased fit (likelihood ratio test comparing species model to full model: $\chi_{1}^{2}=27.05, P<0.001$ ).

Overall, this full model revealed main effects of safe value, condition, species and food preference (see Table 2). This again indicates that individuals had a greater propensity to choose the risky option (1) when the value of the safe alternative was lower, (2) when faced with competition and (3) if they were chimpanzees. While there was no evidence for learning or satiation effects, individual variation in food preferences also predicted overall propensity to choose the risky option. That is, individuals whose preferences skewed more towards the risky outcomes chose the risky option more frequently, and individuals whose preferences skewed towards the safe option chose it more frequently. However, we found no overall difference between the two species in food preferences, and individual differences in preferences would equally affect responses in both the competitive and neutral contexts. Moreover, we found that both condition and species influenced choices, even when controlling for individual variation

Table 2

Factors influencing the likelihood of chimpanzees and bonobos choosing the risky option in study 1 (competition)

\begin{tabular}{lclrl}
\hline Factor & Estimate & \multicolumn{1}{l}{ SE } & \multicolumn{1}{l}{$Z$} & $P$ \\
\hline Condition & 0.759 & 0.146 & 5.181 & $<\mathbf{0 . 0 0 0 1}$ \\
Species & 1.236 & 0.334 & 3.701 & $\mathbf{< . 0 0 0 1}$ \\
Safe value & -1.513 & 0.151 & -9.989 & $<\mathbf{0 . 0 0 0 1}$ \\
Relative food preference & 2.765 & 1.088 & 2.542 & $\mathbf{< 0 . 0 5}$ \\
Trial & -0.001 & 0.016 & -0.077 & 0.939 \\
\hline
\end{tabular}

The full generalized linear mixed model assessed the influence of the following factors: condition (competition versus neutral context), safe value (one versus three pieces of food delivered by the safe option), species (chimpanzees versus bonobos), relative food preference (higher values indicate more relative preference for the risk food types compared to the safe food type) and trial number (1-16 in the session). in food preferences. Together, these findings indicate that condition and species individually explained a significant amount of the variance observed in the apes' patterns of choices between the risky and safe options.

These results support our main hypotheses. First, chimpanzees were more risk prone than bonobos overall: only chimpanzees chose the risky option above chance levels, and they did so at higher rates than bonobos. Second, social context shifted apes' choices, as in humans: apes were more likely to choose the risky option in the competitive context relative to the neutral context. Importantly, our additional analyses indicate that several alternative explanations cannot account for these results. First, we found no overall difference between the two species in preferences for the food items used in the task, and the GLMM results further controlled for individual differences in relative preferences. Second, performance on the risk control trials indicated no difference between the two species in any control type, and apes were extremely successful on the attention controls that were interspersed within both test sessions regardless of condition. As these control trials were structurally identical to risky choice trials, this suggests that differences in attention, memory or overall task comprehension cannot account for our results. However, while social context affected the apes' risk strategies, it is unclear whether the competition context in particular was important, or whether any social interaction would have a similar effect. In study 2 we therefore addressed this possibility.

\section{STUDY 2: DOES PLAY INFLUENCE APE RISK PREFERENCES?}

To assess whether apes' risk preferences would shift in response to any social interaction more generally, we conducted a second experiment involving a different social interaction. Here we compared apes' risk preferences in a positive context (play) to a neutral context. We used a play context because there is some evidence that positive emotions can increase risk-seeking behaviour in humans (Isen \& Patrick 1983; Lerner \& Keltner 2001). That is, if this contextual manipulation impacts risk decision making, we 
predicted it should result in similar shifts towards risk proneness in apes. In contrast, it may be that competition in particular is important in shaping apes' risk preferences. In that case, apes may not show any change in risk preferences in response to this more positive social interaction.

\section{Methods}

\section{Subjects}

We tested 34 apes from the same populations: 20 chimpanzees ( 7 females and 13 males; mean age 11.3 years; range $7-21$ years) and 14 bonobos ( 4 females and 10 males; mean age 8.2 years; range $6-11$ years). All apes had participated in study 1 approximately $2-4$ weeks previously. Two additional bonobos from study 1 did not participate in the second study (see Supplementary Table S1).

\section{Procedure}

Apes completed two test sessions (play condition and neutral condition) in counterbalanced order. All aspects of the task were identical to those in study 1, with the exception of the manner in which E2 interacted with the apes. Here, the neutral condition was identical to that in study 1 . However, in the play condition, E2 played with the ape (in contrast to the competition condition from study 1). In particular, E2 tickled the ape with straw through the bars or mesh, played chase and clapped while laughing. The same experimenters from study 1 played the role of E1 (conducting the risk task) and E2 (interacting with the ape) across all subjects for both studies. That is, the same experimenter who conducted the risk task in study 1 also did so in study 2 . As all subjects had recently completed both the food preference pretest and the risk introduction session in study 1 , we did not repeat those sessions for the current study. A second coder blind to the options' side assignment coded $20 \%$ of trials from video with excellent reliability (Cohen's kappa $=1.0$ ). Data analysis was the same as described for study 1.

\section{Results and Discussion}

\section{Control trials and reward history}

As in study 1, we examined performance on control trials and overall reward history to assess whether either of these factors might influence our results. Both species were at ceiling levels of performance on the interspersed attention control trials, with no differences between the two species (see Table 2). As in study 1, we also confirmed that the interaction with E2 in the play condition did not distract the apes. A comparison of performance on control trials revealed no difference across the two conditions (Wilcoxon signedranks test: $T=4, N=34,29$ ties, $Z=-1.34, P=0.18$ ).

We next examined patterns of reward history; one chimpanzee and one bonobo were not included in these analyses because they never chose the risky option in at least one of the conditions. This revealed that apes received the good outcome on $51.2 \pm 0.7 \%$ of trials (one-sample $t$ test: $t_{31}=1.58, P=0.12$ ). A repeated measures ANOVA revealed no effect of either species or condition on the percentage of trials where apes received the good outcome $(P>0.47$, in both cases). Thus, differences in reward history are unlikely to explain differences in the apes' patterns of risky choice.

\section{Risk preferences}

We first examined choices for the risky option across species (see Fig. 2a). Collapsing across all trials, the chimpanzees chose the risky option on $71.7 \pm 5.6 \%$ of trials, significantly above chance (one-sample $t$ test: $t_{19}=3.89, P=0.001$ ), whereas the bonobos chose the risky option on only $56.6 \pm 6.5 \%$ of trials $\left(t_{13}=1.01\right.$, $P=0.33$ ). This aligns with the results from the previous study indicating that chimpanzees are more risk prone than bonobos. In contrast to the findings from study 1 , only 11 individuals showed increases in choices for the risky option in the play condition, and 15 showed decreases (Wilcoxon signed-ranks test: $T=11+, N=34$, 8 ties, $Z=-1.11, P=0.27$; see Table $S 1$ for individual data). A repeated measures ANOVA revealed a main effect of safe value $\left(F_{1,32}=50.43, P<0.001\right)$, with apes choosing the risky option less often as the value of the safe alternative increased, and a strong
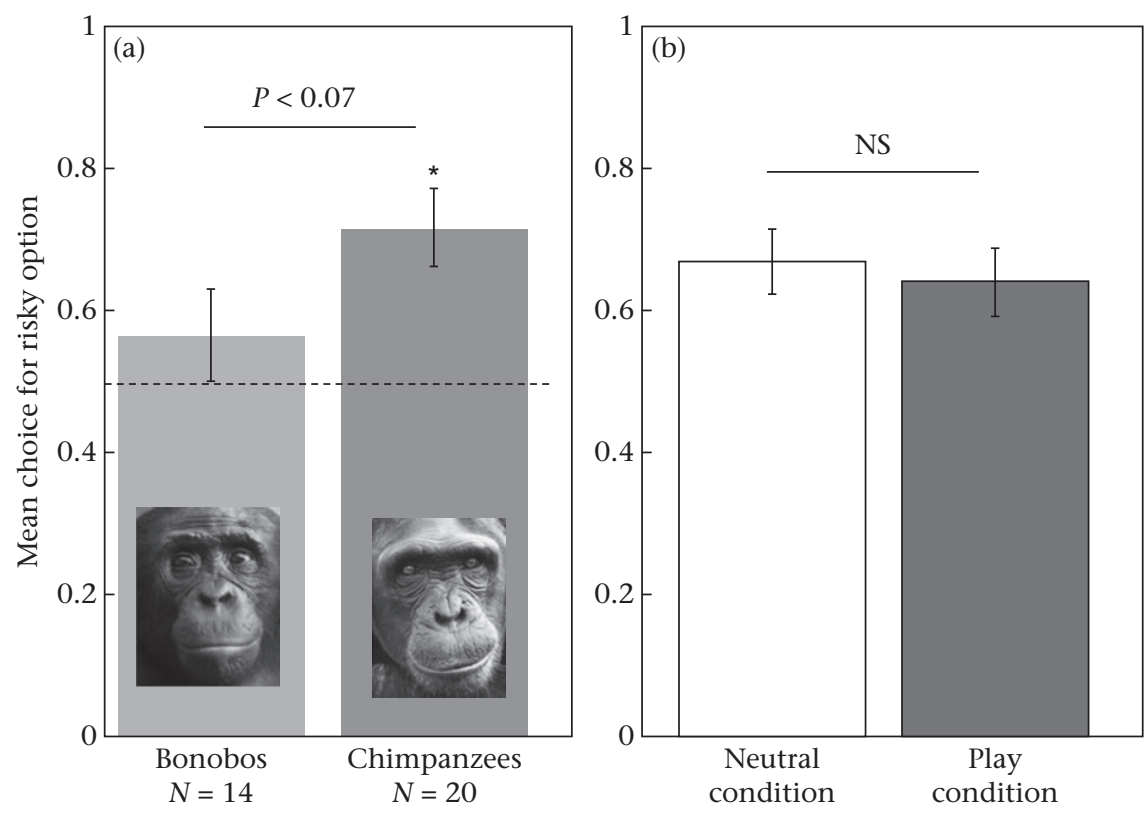

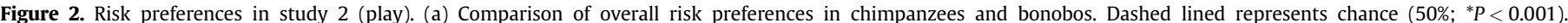

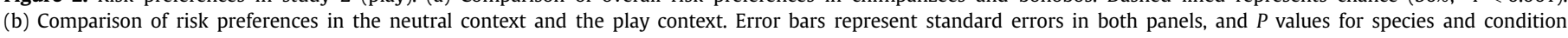
comparisons are based on the repeated measures ANOVA. 
trend for an effect of species $\left(F_{1,32}=3.55, P=0.069\right)$, suggesting that chimpanzees again tended to choose the risky option more than bonobos. However, there was no effect of condition $\left(F_{1,32}=1.04, P=0.32\right)$ : apes chose the risky option on $66.9 \pm 4.6 \%$ of trials in the neutral condition and on $64.0 \pm 4.8 \%$ of trials in the play condition (see Fig. 2b). Finally, there were no significant interactions. Analysing the two species separately revealed that neither independently showed an effect of condition $(P>0.34$, in both cases).

The GLMM analysis confirmed these results. As in study 1, we first fitted a basic model including subject as a random factor, safe value as a fixed factor, and trial number and relative food preference as covariates. Guided by our main hypotheses, we then added condition and species as fixed factors to the more parsimonious basic model to assess whether this increased model fit. Comparing the basic model to a second model that included species as an additional term revealed a significant increase in fit (likelihood ratio test comparing basic to species model: $\chi_{1}^{2}=4.05, P<0.05$ ). A full model that also included condition did not increase fit, however (likelihood ratio test comparing species to full model: $\chi_{1}^{2}=1.54$, $P=0.21$ ). Overall, this full model revealed main effects of safe value, species and food preference, but no effect of condition (see Table 3). Overall, these results replicate our main results indicating that individuals were more likely to choose the risky option (1) when the value of the safe alternative was lower and (2) if they were chimpanzees, even when controlling for the effect of individual differences in food preferences. However, there was no support for a main effect of condition, indicating that the play context did not influence the apes' preferences relative to a neutral context. Together, these findings indicate that while species explains a significant amount of the variance in choices, condition does not.

\section{Comparisons of studies 1 and 2}

Finally, we assessed whether the difference in results between the two studies was due to a global shift in the apes' preferences. Examining the 34 individuals who completed both studies revealed no overall change in choices for risk between studies 1 and 2 $\left(t_{33}=-0.42, P=0.67\right)$. In addition, an analysis of the four conditions that the apes completed across both studies (competition, neutral 1, play, neutral 2; note: that there was an order confound) replicated the main effects of species $\left(F_{1,32}=8.36, P<0.01\right)$, safe value $\left(F_{1,32}=84.52, \quad P<0.001\right)$, and condition $\left(F_{3,96}=3.10\right.$, $P<0.05)$. Post hoc tests showed that there was a significant difference between the competition condition and the first neutral condition (Tukey test: $P<0.05$ ), but no other conditions differed. Overall, this suggests that the different results across the two studies are unlikely to be due to some global change in apes' preferences between the two studies.

Table 3

Factors influencing the likelihood of chimpanzees and bonobos choosing the risky option in study 2 (play)

\begin{tabular}{lclcc}
\hline Factor & Estimate & SE & $Z$ & $P$ \\
\hline Condition & -0.215 & 0.172 & -1.250 & 0.211 \\
Species & 1.217 & 0.595 & 2.05 & $\mathbf{< 0 . 0 5}$ \\
Safe value & -2.611 & 0.198 & -13.184 & $\mathbf{< 0 . 0 0 1}$ \\
Relative food preference & 9.270 & 2.401 & 3.862 & $\mathbf{< 0 . 0 0 1}$ \\
Trial & -0.035 & 0.019 & -1.877 & 0.061 \\
\hline
\end{tabular}

The full generalized linear mixed model assessed the influence of the following factors: condition (play versus neutral context), safe value (one versus three pieces of food delivered by the safe option), species (chimpanzees versus bonobos), relative food preference (higher values indicate more relative preference for the risk food types compared to the safe food type) and trial number (1-16 in the session).

\section{GENERAL DISCUSSION}

These studies provide evidence for two major conclusions concerning the impact of social context on risky decision making in Pan. First, social context can modulate risk preferences in apes: like humans, both chimpanzees and bonobos became more risk prone following a competitive interaction. Even though chimpanzees and bonobos differed in their overall response to risk, as chimpanzees preferred the risky option more than bonobos did, both species were more risk prone in the competitive context than in a neutral context. Results from our control trials further indicated that this change was not due to distraction, as apes had high levels of performance across conditions. Second, the results from study 2 indicated that not all social contexts influence risk preferences in a similar fashion: play did not shift apes' risk preferences relative to a neutral condition. The pattern of results across the two studies therefore suggests that only certain types of social contexts, such as competition, may be important in shaping apes' decision-making strategies.

An important question for future research is why only some types of social interactions influence apes' attitudes towards risk. From an ultimate perspective, competition may be a particularly relevant situation for decisions concerning resources such as food. Competition is a ubiquitous problem for social-living species such as chimpanzees and bonobos that must compete for food when foraging in groups, as well as compete with others for access to mates. Status relative to competitors is also an important predictor of access to both types of resources in apes (Parish 1996; Constable et al. 2001; Wittig \& Boesch 2003b; Surbeck et al. 2011). Thus, apes may have viewed the human competitor as a threat to their resources, and the competitive condition thus would reflect a meaningful social interaction in terms of making economic decisions about food. In contrast, the play interaction may not have been particularly relevant to either resource accumulation or social status. This interpretation aligns with evidence from studies of social cognition suggesting that many species are highly attuned to the threat of competition, compared to more cooperative motives. From a mechanistic perspective, the competition and play contexts may have differed in terms of their psychological impact on the apes. In particular, the contrast in valence between study 1 (involving a more negative competitive context) and study 2 (involving a more positive play contest) suggests that only particular emotional or motivation states are important in shaping an ape's decision making. That is, any negative experience might produce risk-prone behaviours in the apes, regardless of whether they involve competition. Notably, some theoretical views have proposed that competition influences social cognitive skills by altering motivation or emotional states (Hare \& Tomasello 2004), and some studies suggest that emotional reactivity can constrain cooperative abilities in apes (Melis et al. 2006b, c; Hare et al. 2007).

Indeed, research on the role of emotion on decision making in humans indicates that many different emotions can have a profound effect on risk preferences (Loewenstein et al. 2001; Loewenstein \& Lerner 2003). People experience emotions like disappointment and regret in response to the outcomes of their decisions (Coricelli et al. 2007), and studies of individuals with brain lesions suggest a causal role for emotions in shaping risky choice (Camille et al. 2004; Hsu et al. 2005). Most relevant to the current studies, risk preferences can be directly manipulated by inducing mood states such as happiness (Isen \& Patrick 1983), sadness or anxiety (Raghunathan \& Pham 1999), anger or disgust (Fessler et al. 2004), and stress (Porcelli \& Delgado 2009). Previous work in humans has linked competition specifically to anger states, and inducing anger can increase risk-prone decision making (Fessler et al. 2004). Some experimental evidence further suggests 
that competition can directly affect risk preferences by modulating emotions, a shift that can be indexed by physiological measures (Bault et al. 2008). Importantly, recent research indicates that chimpanzees have complex emotional repertoires (Parr et al. 2005). Future research on decision making in apes could therefore examine whether emotional processes modulate the types of social context effects seen here. If such emotional states play a mechanistic role in shaping their decisions about risk, then explicitly inducing emotional states should have a similar impact on their choices.

One surprising aspect of our results is that both chimpanzees and bonobos showed similar responses to the competitive social interaction. Despite their recent divergence, estimated at less than 1 million years ago from genetic data (Won \& Hey 2005), chimpanzees and bonobos show important differences in social systems. In particular, chimpanzees show more sexual dimorphism, more extreme levels of aggression and less sociosexual behaviours than bonobos (Kano 1992; Parish \& de Waal 2000; Hare et al. 2012). One hypothesis is that different levels of scramble competition for food may account for this suite of changes (Wrangham 2000; Wrangham \& Pilbeam 2001). For example, wild chimpanzees may face more competition for smaller, less abundant food patches than do bonobos (White \& Wrangham 1988) and have less access to terrestrial herbaceous vegetation as fallback food (Malenky \& Wrangham 1993). In terms of aggression, chimpanzees show more linear dominance hierarchies and more severe (and sometimes lethal) aggression than do bonobos (Kano 1992; Watts \& Mitani 2001; Boesch et al. 2002; Muller et al. 2009). Experimental comparisons further indicate that bonobos are more able to share food and tolerantly co-feed when than are chimpanzees (Hare et al. 2007; Wobber et al. 2010a, b). One prediction from this set of observational and experimental results is that chimpanzees might respond more strongly to the threat of competition than bonobos. Similarly, ringtailed lemurs, which have more complex social structures involving linear dominance hierarchies, show different reactions to competitors compared to other lemur species (Sandel et al. 2011). However, our results indicate that both species showed similar responses to the particular social context manipulation used in the current studies.

What can account for this pattern of results? One possible explanation is that the current study involved a human competitor, not conspecifics. Observations of these species in the wild obviously involve conspecific interactions, as do several experimental studies involving conspecific partners (Hare et al. 2007; Wobber et al. 2010a, b). Importantly, these types of observations and experiments cannot equate the behaviour of social partners across species, and are indeed not designed to do so, as their goal is to understand naturalistic social interactions. In contrast to interactions with conspecifics, the human competitor in the current study showed a predetermined behavioural repertoire in response to both species. While human competitors have been used in several studies involving chimpanzees (Hare \& Tomasello 2004; Hare et al. 2006; Melis et al. 2006a; Kaminski et al. 2008), there are few such studies involving bonobos (but see Herrmann \& Tomasello 2006). Thus, an important goal for future research is to assess how the threat of conspecific competition may impact these species' risk preferences. This type of study might reveal that chimpanzees show a more pronounced reaction to conspecific competition than do bonobos.

An alternative possibility, however, is that while chimpanzees and bonobos showed the same behavioural response to competition in our study, the underlying psychological mechanisms may differ. Importantly, diverse emotional experiences can induce similar changes in risk taking in humans (Isen \& Patrick 1983; Raghunathan \& Pham 1999; Lerner \& Keltner 2001; Fessler et al. 2004; Porcelli \& Delgado 2009). Therefore, apes may show similar patterns of risk-prone decision making in competitive contexts, despite different underlying psychological reactions to the social interaction. An analogous phenomenon occurs in these species' hormonal responses to conspecific competition. In particular, while both species show anticipatory changes in steroid hormones prior to interactions involving food, bonobos show these shifts in cortisol, whereas chimpanzees show similar shifts in testosterone levels (Wobber et al. 2010a). That is, while the behavioural response to competition was similar, the neurohormonal mechanisms underpinning the responses varied across the two species.

Overall, the current results contribute to the growing evidence that many decision-making biases seen in humans are shared with other species: humans, chimpanzees and bonobos all show a propensity to respond to competitive interactions with more riskprone decision-making styles. Indeed, the myriad evidence for context-dependent decision making in animals suggests that many anomalies or cognitive biases that puzzle economists are in fact not unique to humans. Rather, they are shared with many taxonomically diverse species, including insects, birds, rodents and primates. While many researchers have begun to apply evolutionary principles to economic decision making in humans (e.g. Fessler et al. 2004; Wilson \& Daly 2004; Ermer et al. 2008; Hill \& Buss 2010), studies of humans alone can only go so far. Indeed, one of the most powerful tools in evolutionary biology is the comparative method: examining the traits of different populations or species that have been shaped by differing ecological or social forces in order to better understand how natural selection proceeded (Clutton-Brock \& Harvey 1979; Mayr 1982). Consequently, placing human decision making in a broader comparative framework, including both apes and more distant taxa, is critical to illuminating the evolutionary origins of economic behaviour.

\section{Acknowledgments}

We thank Kara Schroepfer with assistance in conducting the studies, and Kerri Rodriguez and Ashton Madison for assistance with coding. Felix Warneken and Victoria Wobber gave welcome comments on an earlier version of the manuscript. At Tchimpounga Chimpanzee Sanctuary, we thank Rebeca Atencia, Debby Cox, Lisa Pharoah, Keith Brown and Jane Goodall Institute USA in collaboration with the Congolese Ministere de la Recherche Scientifique et de l'Innovation Technique for hosting our research. We appreciate the assistance of the animal caretakers, especially Jean Maboto and Bernard Moumbaka. At Lola ya Bonobo, we thank Claudine Andre, Dominique Morel, Fanny Mehl, and Pierrot Mbonzo for their support in collaboration with the Ministry of Research and the Ministry of Environment in the Democratic Republic of Congo for supporting our research (research permit: MIN.RS/SG/004/2009). We appreciate the assistance of the animal caregivers, especially John Claude Nzumbi, Stany Mokando and Philippe Kunaka. This work was supported in part by European Research Commission Advanced Grant Agreement 233297 and National Science Foundation grants NSF-BCS-08-27552-02 and NSF-BCS-10-25172 to B.H.

\section{Supplementary Material}

Supplementary material for this article is available, in the online version, at http://dx.doi.org/10.1016/j.anbehav.2012.07.010.

\section{References}

Baayen, R. H. 2008. Analyzing Linguistic Data: a Practical Introduction to Statistics. Cambridge, Massachusetts: Cambridge University Press.

Bates, D. 2010. Ime4 Package: Linear Mixed-effects Models Using S4 Classes. http:// www.R-project.org. 
Bault, N., Coricelli, G. \& Rustichini, A. 2008. Interdependent utilities: how social ranking affects choice behavior. PLOS ONE, 3, e3477, http://dx.doi.org/10.1371/ journal.pone.0003477.

Boesch, C., Hohmann, G. \& Marchant, L. F. 2002. Behavioral Diversity in Chimpanzees and Bonobos. Cambridge: Cambridge University Press.

Bolker, B. M., Brooks, M. E., Clark, C. J., Geange, S. W., Poulsen, J. R., Stevens, M. H. H. \& White, J. S. S. 2008. Generalized linear mixed models: a practical guide for ecology and evolution. Trends in Ecology \&' Evolution, 24, 127-135.

Bugnyar, T. \& Heinrich, B. 2005. Ravens, Corvus corax, differentiate between knowledgeable and ignorant competitors. Proceedings of the Royal Society $B$, 272, 1641-1646.

Bugnyar, T., Schwab, C., Schloegl, C., Kotrschal, K. \& Heinrich, B. 2007. Ravens judge competitors through experience with play caching. Current Biology, 17, 1804-1808.

Burkhart, J. \& Heschl, A. 2007. Understanding visual access in common marmosets, Callithrix jacchus: perspective taking or behavior reading? Animal Behaviour, 73. 457-469.

Byrne, R. W. \& Whiten, A. 1988. Machiavellian Intelligence: Social Expertise and the Evolution of Intellect In Monkeys, Apes, and Humans. Oxford: Oxford University Press.

Call, J., Hare, B., Carpenter, M. \& Tomasello, M. 2004. 'Unwilling' versus 'unable': chimpanzees' understanding of human intentional action. Developmental Science, 7, 488-498.

Camille, N., Coriecelli, G., Sallet, J., Pradat-Diehl, P., Duhamel, J. R. \& Sirigu, A. 2004. The involvement of the orbitofrontal cortex in the experience of regret. Science, 304, 1167-1170.

Caraco, T. 1981. Energy budgets, risk, and foraging preferences in dark-eyed juncos (Junco hyemalis). Behavioral Ecology and Sociobiology, 8, 213-217.

Clayton, N. S., Dally, J. M. \& Emery, N. J. 2007. Social cognition by food-caching corvids: the western scrub-jay as a natural psychologist. Philosophical Transactions of the Royal Society B, 362, 507-522.

Clutton-Brock, T. H. \& Harvey, P. H. 1979. Comparison and adaptation. Proceedings of the Royal Society $B, \mathbf{2 0 5}, 547-565$.

Constable, J. L., Ashley, M. V., Goodall, J. \& Pusey, A. E. 2001. Noninvasive paternity assignment in Gombe chimpanzees. Molecular Ecology, 10, 1279-1300.

Coricelli, G., Dolan, R. J. \& Sirigu, A. 2007. Brain, emotion, and decision making: the paradigmatic example of regret. Trends in Cognitive Sciences, 11, 258-265.

Dally, J. M., Emery, N. J. \& Clayton, N. S. 2004. Cache protection strategies by western scrub-jays (Aphelocoma californica): hiding food in the shade. Proceedings of the Royal Society B, Supplement, 271, S387-S390.

Dally, J. M., Emery, N. J. \& Clayton, N. S. 2006. Food-caching western scrub jays keep track of who was watching when. Science, 312, 1662-1665.

Emery, N. J. \& Clayton, N. S. 2001. Effects of experience and social context on prospective caching strategies by scrub jays. Nature, 414, 443-446.

Ermer, E., Cosmides, L. \& Tooby, J. 2008. Relative status regulated risky decision making about resources in men: evidence for the co-evolution of motivation and cognition. Evolution and Human Behavior, 29, 106-118.

Fessler, D. M. T., Pilsworth, E. G. \& Flamson, T. J. 2004. Angry men and disgusted women: an evolutionary approach to the influence of emotions on risk-taking. Organizational Behavior and Human Decision Processes, 95, 107-123.

Flombaum, J. I. \& Santos, S. 2005. Rhesus monkeys attribute perceptions to others. Current Biology, 15, 447-452.

Gilby, I. C. \& Wrangham, R. W. 2007. Risk-prone hunting by chimpanzees (Pan troglodytes schweinfurthii) increases during periods of high diet quality. Behavioral Ecology and Sociobiology, 61, 1771-1779.

Glass, B. D., Maddox, W. T., Bowen, C., Savarie, Z. R., Matthews, M. D., Markman, A. B. \& Schnyer, D. M. 2011. The effects of 24-hour sleep deprivation on the exploration-exploitation trade-off. Biological Rhythm Research, 42, 99-110.

Hare, B. 2001. Can competitive paradigms increase the validity of experiments on primate social cognition? Animal Cognition, 4, 269-280.

Hare, B. \& Tomasello, M. 2004. Chimpanzees are more skilful in competitive than in cooperative tasks. Animal Behaviour, 68, 571-581.

Hare, B., Addessi, E., Call, J., Tomasello, M. \& Visalberghi, E. 2003. Do capuchin monkeys, Cebus apella, know what conspecifics do and do not see? Animal Behaviour, 65, 131-142.

Hare, B., Call, J. \& Tomasello, M. 2006. Chimpanzees deceive a human competitor by hiding. Cognition, 101, 495-514.

Hare, B., Melis, A., Woods, V., Hastings, S. \& Wrangham, R. 2007. Tolerance allows bonobos to outperform chimpanzees on a cooperative task. Current Biology, 17, 619-623.

Hare, B., Wobber, V. \& Wrangham, R. 2012. The self-domestication hypothesis: evolution of bonobo psychology is due to selection against aggression. Animal Behaviour, 83, 573-585.

Haun, D. B. M., Nawroth, C. \& Call, J. 2011. Great apes' risk-taking strategies in a decision making task. PLoS ONE, 6, e28801.

Heilbronner, S. H., Rosati, A. G., Stevens, J. R., Hare, B. \& Hauser, M. 2008. A fruit in the hand or two in the bush? Divergent risk preferences in chimpanzees and bonobos. Biology Letters, 4, 246-249.

Herrmann, E. \& Tomasello, M. 2006. Apes' and children's understanding of cooperative and competitive motives in a communicative situation. Developmental Science, 9, 518-529.

Herrmann, E., Call, J., Hernandez-Lloreda, M. V., Hare, B. \& Tomasello, M. 2007. Humans have evolved specialized skills of social cognition: the cultural intelligence hypothesis. Science, 317, 1360-1366.
Herrmann, E., Hare, B., Call, J. \& Tomasello, M. 2010. Differences in the cognitive skills of bonobos and chimpanzees. PLOS ONE, 5, e12438.

Hill, S. E. \& Buss, D. M. 2010. Risk and relative social rank: positional concerns and risky shifts in probabilistic decision-making. Evolution and Human Behavior, 31 219-226.

Hsu, M., Bhatt, M., Adolphs, R., Tranel, D. \& Camerer, C. 2005. Neural systems responding to degrees of uncertainty in human decision-making. Science, $\mathbf{3 1 0}$ 1680-1683.

Isen, A. M. \& Patrick, R. 1983. The effect of positive feelings on risk taking: when the chips are down. Organizational Behavior and Human Performance, 31 194-202

Kahneman, D. \& Tversky, A. 2000. Choices, Values, and Frames. New York: Cambridge University Press.

Kaminski, J., Call, J. \& Tomasello, M. 2008. Chimpanzees know what others know but not what they believe. Cognition, 109, 224-234.

Kano, T. 1992. The Last Ape: Pygmy Chimpanzee Behavior and Ecology. Stanford, California: Stanford University Press.

Kassam, K., Koslov, K. \& Mendes, W. B. 2009. Decisions under distress: stress profiles influence anchoring and adjustment. Psychological Science, 20, 1394-1399.

Lakshminarayanan, V. R., Chen, M. K. \& Santos, L. R. 2011. The evolution of decision-making under risk: framing effects in monkey risk preferences. Journal of Experimental Social Psychology, 47, 689-693.

Lerner, J. S. \& Keltner, D. 2001. Fear, anger, and risk. Journal of Personality and Social Psychology, 81, 146-159.

Loewenstein, G. F. \& Lerner, J. S. 2003. The role of affect in decision making. In: Handbook of Affective Science (Ed. by R. J. Davidson, K. R. Scherer \& H. H. Goldsmith), pp. 619-642. Oxford: Oxford University Press.

Loewenstein, G. F., Weber, E. U., Hsee, C. K. \& Welch, N. 2001. Risk as feelings Psychological Bulletin, 127, 267-286.

Malenky, R. K. \& Wrangham, R. W. 1993. A quantitative comparison of terrestrial herbaceous food consumption by Pan paniscus in the Lomako Forest, Zaire, and Pan troglodytes in the Kibale Forest, Uganda. American Journal of Primatology, 32,1-12.

Marsh, B. \& Kacelnik, A. 2002. Framing effects and risky decisions in starlings. Proceedings of the National Academy of Sciences, U.S.A., 99, 3352-3355.

Mayr, E. 1982. The Growth of Biological Thought. Cambridge, Massachusetts: Harvard University Press.

Melis, A., Call, J. \& Tomasello, M. 2006a. Chimpanzees (Pan troglodytes) conceal visual and auditory information from others. Animal Behaviour, 120, 154-162.

Melis, A., Hare, B. \& Tomasello, M. 2006b. Chimpanzees recruit the best collaborators. Science, 311, 1297-1300.

Melis, A., Hare, B. \& Tomasello, M. 2006c. Engineering cooperation in chimpanzees: tolerance constraints on cooperation. Animal Behaviour, 72, 275-286.

Mitani, J. C., Watts, D. P. \& Amsler, S. J. 2010. Lethal intergroup aggression leads to territorial expansion in wild chimpanzees. Current Biology, 20, R507-R508.

Muller, M., Kahlenberg, S. M. \& Wrangham, R. 2009. Male aggression agains females and sexual coercion in chimpanzees. In: Sexual Coercion in Primates and Humans: an Evolutionary Perspective on Male Aggression against Females (Ed. by M. Muller \& R. Wrangham), pp. 3-22. Cambridge, Massachusetts: Harvard University Press.

Parish, A. R. 1996. Female relationships in bonobos (Pan paniscus): evidence for bonding, cooperation, and female dominance in a male-philopatric species. Human Nature, 7, 61-96.

Parish, A. R. \& de Waal, F. B. M. 2000. The other 'closest living relative': how bonobos (Pan paniscus) challenge traditional assumptions about females, dominance, intra- and intersexual interactions, and hominid evolution. Annals of the New York Academy of Sciences, 907, 97-113.

Parr, L. A., Waller, B. M. \& Fugate, J. 2005. Emotional communication in primates: implications for neurobiology. Current Opinion in Neurobiology, 15, 1-5.

Porcelli, A. J. \& Delgado, M. R. 2009. Acute stress modulates risk taking in financial decision making. Psychological Science, 20, 278-283.

Pusey, A., Williams, J. \& Goodall, J. 1997. The influence of dominance rank on the reproductive success of female chimpanzees. Science, 277, 828-831.

R Development Core Team 2011. A Language and Environment for Statistical Computing. Vienna, Austria: R Foundation for Statistical Computing. http:// www.R-project.org.

Raghunathan, R. \& Pham, M. T. 1999. All negative moods are not equal: motivational influences of anxiety and sadness on decision making. Organizational Behavior and Human Decision Processes, 79, 56-77.

Reynolds, B. \& Schiffbauer, R. 2004. Measuring state changes in human delay discounting: an experiential discounting task. Behavioural Processes, 67, 343-356.

Rosati, A. G. \& Stevens, J. R. 2009. Rational decisions: the adaptive nature of context-dependent choice. In: Rational Animals, Irrational Humans (Ed. by A. P. Watanabe, L. Blaisdell, L. Huber \& A. Young), pp. 101-117. Tokyo: Keio University Press.

Rosati, A. G. \& Hare, B. 2011. Chimpanzees and bonobos distinguish between risk and ambiguity. Biology Letters, 7, 15-18.

Sandel, A. A., MacLean, E. L. \& Hare, B. 2011. Evidence from four lemur species that ringtailed lemur social cognition converges with that of haplorhine primates. Animal Behaviour, 81, 925-931.

Santos, L. R., Nissen, A. G. \& Ferrugia, J. A. 2006. Rhesus monkeys, Macaca mulatta, know what others can and cannot hear. Animal Behaviour, 71, 1175-1181.

van Schaik, C. P. \& van Hooff, J. A. R. A. M. 1983. On the ultimate causes of primate social systems. Behaviour, 85, 91-117. 
Schuck-Paim, C., Pompilio, L. \& Kacelnik, A. 2004. State-dependent decisions cause apparent violations of rationality in animal choice. PLoS Biology, 2, e402.

Seed, A. M., Clayton, N. S. \& Emery, N. J. 2008. Cooperative problem solving in rooks (Corvus frugilegus). Proceedings of the Royal Society B, 275, 1421-1429.

Shafir, S., Waite, T. A. \& Smith, B. H. 2002. Context-dependent violations of rationa choice in honeybees (Apis mellifera) and gray jays (Perisoreus canadensis). Behavioral Ecology and Sociobiology, 51, 180-187.

Sterck, E. H. M., Watts, D. P. \& van Schaik, C. P. 1997. The evolution of female social relationships in nonhuman primates. Behavioral Ecology and Sociobiology, 41, 291-309.

Stevens, J. R., Rosati, A. G., Heilbronner, S. R. \& Muelhoff, N. 2011. Waiting for grapes: expectancy and delayed gratification in bonobos. International Journal of Comparative Psychology, 24, 99-111.

Surbeck, M., Mundry, R. \& Hohmann, G. 2011. Mothers matter! Maternal support, dominance status and mating success in male bonobos (Pan paniscus). Proceedings of the Royal Society B, 278, 590-598.

Tomasello, M., Call, J. \& Hare, B. 2003. Chimpanzees understand psychological states: the question is which ones and to what extent. Trends in Cognitive Sciences, 7, 153-156.

Venkatraman, V., Huettel, S. A., Chuah, L. Y. M., Payne, J. W. \& Chee, M. W. L. 2011 Sleep deprivation biases the neural mechanisms underlying economic preferences. Journal of Neuroscience, 31, 3712-3718.

de Waal, F. B. M. 1982. Chimpanzee Politics: Power and Sex among Apes. New York: Harper \& Row.

Waite, T. A. 2001. Intransitive preferences in hoarding gray jays (Perisoreus canadensis). Behavioral Ecology and Sociobiology, 50, 116-121.

Watts, D. P. \& Mitani, J. C. 2001. Boundary patrols and intergroup encounters in wild chimpanzees. Behaviour, 138, 299-327.

Werdenich, D. \& Huber, L. 2002. Social factors determine cooperation in marmosets. Animal Behaviour, 64, 771-781.

White, F. J. \& Wrangham, R. W. 1988. Feeding competition and patch size in the chimpanzee species Pan paniscus and Pan troglodytes. Behaviour, 105, 148-164.
Wilson, M. \& Daly, M. 2004. Do pretty women inspire men to discount the future? Proceedings of the Royal Society B, Supplement, 271, 177-179.

Wittig, R. M. \& Boesch, C. 2003a. 'Decision-making' in conflicts of wild chimpanzees (Pan troglodytes): an extension of the Relational Model. Behavioral Ecology and Sociobiology, 55, 209.

Wittig, R. M. \& Boesch, C. 2003b. Food competition and linear dominance hierarchy among female chimpanzees of the Tai National Park. International Journal of Primatology, 24, 847-867.

Wobber, V. \& Hare, B. 2011. Psychological health of orphan bonobos and chimpanzees in African sanctuaries. PLOS ONE, 6, e17147.

Wobber, V., Hare, B., Maboto, J., Lipson, S., Wrangham, R. \& Ellison, P. T. 2010a. Differential changes in steroid hormones before competition in bonobos and chimpanzees. Proceedings of the National Academy of Sciences, U.S.A., 107, 12457-12462.

Wobber, V., Wrangham, R. \& Hare, B. 2010b. Bonobos exhibit delayed developmental of social behavior and cognition relative to chimpanzees. Current Biology, 20, 226-230.

Won, Y. J. \& Hey, J. 2005. Divergence population genetics of chimpanzees. Molecular Biology and Evolution, 22, 297-307.

Wrangham, R. W. 1980. An ecological model of female-bonded primate groups. Behaviour, 75, 262-300.

Wrangham, R. W. 2000. Why are male chimpanzees more gregarious than mothers? A scramble competition hypothesis. In: Primate Males: Causes and Consequences of Variation in Group Composition (Ed. by P. Kappeler), pp. 248-258. Cambridge: Cambridge University Press.

Wrangham, R. \& Pilbeam, D. 2001. African apes as time machines. In: All Apes Great and Small. Vol. I: African Apes (Ed. by B. M. F. Galdikas, N. E. Briggs, L. K. Sheeran, G. L. Shapiro \& J. Goodall), pp. 5-18. New York: Kluwer Academic.

Yamamoto, S. \& Tanaka, M. 2009. The influence of kin relationship and reciprocal context on chimpanzees' other-regarding preferences. Animal Behaviour, 79 , 595-602. 